Article

\title{
Hierarchical Scheduling Scheme for AC/DC Hybrid Active Distribution Network Based on Multi-Stakeholders
}

\author{
Chang Ye, Shihong Miao *, Yaowang Li, Chao Li and Lixing Li \\ State Key Laboratory of Advanced Electromagnetic Engineering and Technology, Hubei Electric Power Security \\ and High Efficiency Key Laboratory, School of Electrical and Electronic Engineering, Huazhong University of \\ Science and Technology, Wuhan 430074, China; seee_yechang@hust.edu.cn (C.Y.); yw_li@hust.edu.cn (Y.L.); \\ 1x_lichao47@hust.edu.cn (C.L.); D201577339@hust.edu.cn (L.L.) \\ * Correspondence: shmiao@hust.edu.cn; Tel.:+86-13971604685
}

Received: 4 September 2018; Accepted: 17 October 2018; Published: 19 October 2018

check for updates

\begin{abstract}
This paper presents a hierarchical multi-stage scheduling scheme for the AC/DC hybrid active distribution network (ADN). The load regulation center (LRC) is considered in the developed scheduling strategy, as well as the AC and DC sub-network operators. They are taken to be different stakeholders. To coordinate the interests of all stakeholders, a two-level optimization model is established. The flexible loads are dispatched by LRC in the upper-level optimization model, the objective of which is minimizing the loss of the entire distribution network. The lower-level optimization is divided into two sub-optimal models, and they are carried out to minimize the operating costs of the AC/DC sub-network operators respectively. This two-level model avoids the difficulty of solving multi-objective optimization and can clarify the role of various stakeholders in the system scheduling. To solve the model effectively, a discrete wind-driven optimization (DWDO) algorithm is proposed. Then, considering the combination of the proposed DWDO algorithm and the YALMIP toolbox, a hierarchical optimization algorithm (HOA) is developed. The HOA can obtain the overall optimization result of the system through the iterative optimization of the upper and lower levels. Finally, the simulation results verify the effectiveness of the proposed scheduling scheme.
\end{abstract}

Keywords: AC/DC hybrid active distribution; hierarchical scheduling; multi-stakeholders; discrete wind driven optimization

\section{Introduction}

The active distribution network (ADN) is considered an effective way to address the issues caused by the large-scale integration of distributed energy resources (DERs). For this reason, it has aroused great attention in both research and industry fields, and a great number of studies have been done [1-4]. With the large-scale integration of distributed generation (DG), flexible loads, energy storage and new types of power electronic control devices, a large amount of DC equipment has been installed in AC distribution networks. Due to the large number of electrical energy conversion links, using AC distribution networks for power supply only will influence both the cost and the efficiency [5]. The best topology that can accommodate both AC and DC technologies with less need for such conversion is a hybrid one [6]. The idea is to merge the $\mathrm{AC}$ and $\mathrm{DC}$ distribution networks through a bidirectional converter and establish an AC/DC hybrid ADN in which AC- or DC-type energy sources and loads can be flexibly integrated into the distribution networks such that power can smoothly flow between them [7]. Furthermore, flexible DC technology is applied to construct the AC/DC hybrid ADN, which can achieve energy scheduling over a wide area. The AC/DC hybrid ADN is controllable and allows 
DC loads and various DERs to plug-and-play easily; it will be an important implementation form of the future distribution network [8].

The characteristics of the AC/DC hybrid ADN provide an efficient means for the integration of DERs with minimum modifications of the current distribution grid [9]. At present, most research on AC/DC hybrid ADNs is focused on the optimal power flow (OPF) problem and coordinated control methods. An efficient OPF algorithm for hybrid AC/DC grids with discrete control devices is presented in [10]. On this basis, the corrective security constraint is taken into account in OPF for a meshed AC/DC power transmission network [11]. Meanwhile, second-order cone programming (SOCP) is applied, and [12] presents a SOCP formulation of the OPF problem for AC/DC systems with voltage source converter (VSC) technology. In terms of coordinated control methods, Ref. [13] presents an overview of various control schemes used for voltage and frequency regulation in standalone and transition mode operation of the hybrid micro-grid. Ref. [14] proposes an instantaneous power-based current control scheme for reactive power compensation in hybrid AC/DC networks, while Ref. [15] presents a novel three-phase reactive power and voltage distributed control method for AC/DC hybrid ADNs based on model predictive control. Furthermore, in order to ensure the safety of the converters and the grid facility, a flexible control strategy is proposed for the AC/DC hybrid grid in [16]. These studies provide strong support for the optimal scheduling and operation of the AC/DC hybrid ADN.

In general, the core technologies for operation of AC/DC hybrid ADN contain flexible DC technology and scheduling technology [17]. Flexible DC technology can provide a better interface for DGs, energy storage, electric vehicles, and other devices [18-20]. Meanwhile, the ADN scheduling technology can flexibly dispatch various types of flexible resources in the distribution network, and ultimately realize the optimal operation of the entire system. Using a hybrid-type distribution system can eliminate several conversion stages and thus improve efficiency and reduce investment costs [21].

Recently, several studies have involved the optimization of the scheduling strategies of the AC/DC hybrid ADN. Ref. [22] builds a system structure for an AC/DC hybrid ADN and then establishes a multi-time scale optimal dispatch model based on a multi-agent system (MAS). The model is designed to reduce the operation cost and energy loss on the premise of absorbing the most DERs. As the AC and DC sub-networks perform power interaction through an interlink convertor (ILC), a hierarchical planning method is introduced into the scheduling process. Ref. [23] focuses on power management in the AC/DC microgrid, and the combination of PV, fuel cells, wind, and battery storage with adjustable parameters is analyzed. An optimization model is investigated to reduce the implementation costs by using a multi-objective particle swarm optimization (MOPSO) algorithm. For an ADN with a single DC section and multiple AC sections, [24] proposes a hierarchical and distributed coordinated multi-source optimal scheduling strategy which divides the dispatch area into a local level and an area level. In [25], a two-stage stochastic centralized dispatch scheme is presented, where the first stage produces day-ahead dispatch decisions for the dispatchable DG units, while the second stage determines appropriate corrective decisions for a set of possible scenarios. On this basis, a robust optimal method is introduced. Reference [26] proposes a bi-level two-stage robust optimal scheduling model for AC/DC hybrid multi-microgrids. In this model, the system is divided into the utility level and the supply level, and two-stage robust optimization is carried out. A multi-interval-uncertainty constrained robust dispatch model is proposed in [27], in order to deal with the uncertainties of renewable energy generation and load power in an AC/DC hybrid microgrid.

The above studies made great efforts towards developing optimal operation models for AC/DC hybrid ADN; however, there are still some persisting problems that need to be addressed. Firstly, as different stakeholders, the AC and DC subgrids should have their own scheduling objectives. Meanwhile, the distribution network operator (DNO) needs to regulate the power grid globally. Few studies consider their scheduling strategy based on multi-stakeholders of an AC/DC hybrid ADN. Secondly, most of the present studies pay little attention to the coordination of various schedulable resources in an AC/DC hybrid ADN. In addition, the interaction between the AC and DC sides still deserves further study. Thus, this paper constructs a coordinated optimization framework for 
AC/DC hybrid ADN based on multi-stakeholders, and a hierarchical multi-stage scheduling model is established. The upper level is optimized by the load regulation center (LRC), taking the minimum loss of the entire distribution network as the scheduling objective. Meanwhile, the lower level is optimized by the AC and DC sub-networks. Therefore, lower-level optimization is divided into two suboptimal problems, which have objective functions related to their own stakeholders. The advantages of using the above hierarchical optimization scheduling model include: (a) avoiding the difficulty of solving multi-objective optimization; and (b) ensuring that all stakeholders pay attention to their own benefits, and obtain the optimal operating state of the system through mutual cooperation and coordination. Meanwhile, considering the combination of the proposed DWDO algorithm and the YALMIP toolbox, the model is solved by a hierarchical optimization algorithm. The simulation results show that the proposed strategy can minimize the operating costs of AC/DC sub-networks while reducing system losses.

In summary, the contributions of this paper are as follows: (a) a hierarchical multi-stage scheduling model for AC/DC hybrid and is proposed, to avoid the difficulty of solving multi-objective optimization; (b) AC and DC sub-networks are regarded as different stakeholders so that their interaction characteristics are analyzed in a scheduling problem; and (c) a hierarchical optimization algorithm comprising the DWDO algorithm and YALMIP toolbox is developed to solve the hierarchical optimization model.

The rest of this paper is organized into the following sections. Section 2 describes the network structure of the AC/DC hybrid ADN and proposes a hierarchical multi-stage scheduling framework based on multi-stakeholders. Section 3 details the mathematical formulation of the hierarchical optimization scheduling model. In Section 4, a hierarchical optimization algorithm is proposed and researched. In Section 5, case studies and discussions are carried out. Finally, Section 6 put forwards the conclusions that can be drawn.

\section{System Framework}

As mentioned above, various types of distributed scheduling resources are integrated into the AC/DC hybrid ADN. When the distribution network is in operation, the schedulable resources will interact with each other. Scheduling only a single or partial resource makes it difficult to achieve overall system optimization, and easily leads to redundancy and waste of resources. Thus, global optimization of AC/DC hybrid ADN is necessary. On the other hand, each of the AC and DC areas constitutes a sub-network, which can be scheduled as an independent stakeholder. In view of this, the network structure is shown in Figure 1. The arrows in the figure show the positive power direction.

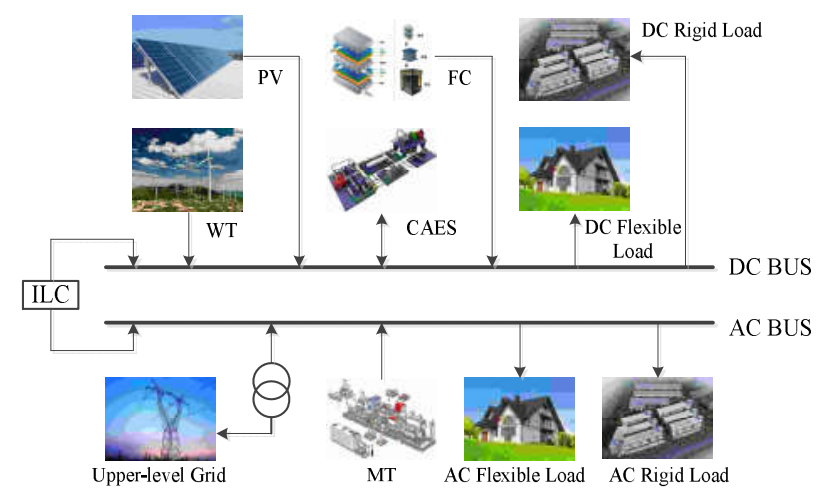

Figure 1. Network structure of the AC/DC hybrid ADN.

In Figure 1, the AC/DC hybrid ADN is divided into an AC section and a DC section. The main components of the DC section include photovoltaics (PVs), wind turbines (WT), fuel cells (FC), compressed air energy storage (CAES), and DC loads, which are connected to the DC bus. The AC section mainly constitutes a micro-gas turbine (MT) and AC loads, with the upper-level power grid 
(UPG) connected to it. The upper-level power grid supplies electricity based on time-of-use price. Meanwhile, the DC section interacts with the AC section through ILC, which can transmit power in both directions. Additionally, in order to reflect the operating characteristics of the ADN, flexible loads are considered to participate in the scheduling.

In general, the power on both the AC and DC sides influences each other, and it is difficult to directly obtain the optimal operating status of the entire system. Thus, a hierarchical multi-stage scheduling framework based on multi-stakeholders is proposed, as shown in Figure 2. It is necessary to consider both active and reactive power in the optimal power flow of the distribution network [28]. However, in this paper, the main purpose of the lower-level optimization is to determine the active economic dispatch of the power system. Meanwhile, considering the complexity of this model, only active power is analyzed in both the AC and DC sections.

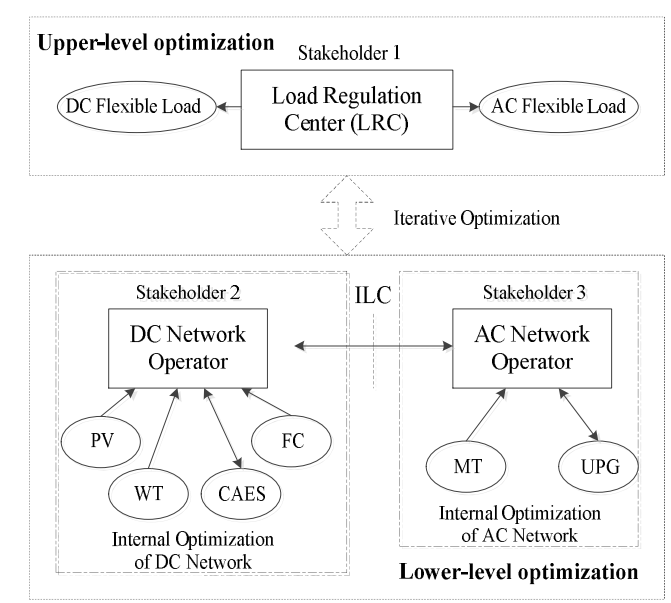

Figure 2. Hierarchical multi-stage scheduling framework based on multi-stakeholders.

In Figure 2, the stakeholder of the upper-level optimization is the load regulation center (LRC). All flexible loads of DC and AC sections are managed by the LRC, in order to reduce the losses of the distribution network. Then, the scheduling results of the flexible loads are transferred to the lower-level optimization, which is comprised of the internal optimization of both the DC network and the AC network. The DGs and CAES are scheduled by the lower-level optimization, as well as the transmission power of the UPG. The results are also transferred to the upper level, and the system performs repeated iterative optimization until it reaches a dynamic balance. Through the hierarchical optimization method, optimal scheduling results for the entire AC/DC hybrid ADN are expected to finally be obtained. The results not only reflect the global optimization of the power grid, but also reflect the local optimization of the AC and DC power grids.

In the next section, we will establish the hierarchical optimization model, and give a detailed solution process for it.

\section{Hierarchical Optimization Model}

\subsection{Optimization Model of the Upper Level}

In the upper-level optimization, flexible loads of the entire network are scheduled. The scheduling objective is to reduce system losses, which mainly include line loss and loss of ILC. Therefore, the objective function is expressed in Equation (1).

$$
\min F_{\mathrm{ul}}=\sum_{t=1}^{T}\left(c_{\mathrm{g}, t} P_{\text {loss } \_, t}+c_{\text {loss } \_I L C} P_{\text {loss } \_I L C, t}\right) \Delta t
$$

where $T$ is the scheduling period. 
The detailed expression of the above power loss can be expressed as Equations (2) and (3).

$$
\begin{gathered}
P_{\text {loss_ }, 1, t}=\sum_{l_{1} \in N_{\text {line }}^{A C}} P_{\text {loss }, l_{1}, t}+\sum_{l_{2} \in N_{\text {line }}^{D C}} P_{\text {loss }, l_{2}, t}=\sum_{l_{1} \in N_{\text {line }}^{A C}} \frac{\left(P_{l_{1}, t}^{2}+Q_{l_{1}, t}^{2}\right) R_{l_{1}}}{U_{\mathbf{e}_{-} A C}^{2}}+\sum_{l_{2} \in N_{\text {line }}^{D C}} \frac{P_{l_{2}, t}^{2} R_{l_{2}}}{U_{\mathbf{e}_{-} D C}^{2} D C} \\
P_{\text {loss_ILC }, t}=\left(1-\eta_{\text {ILC }}\right) \cdot\left|P_{\text {ILC }, t}\right|
\end{gathered}
$$

where $l_{1}$ and $l_{2}$ are lines of the AC and DC networks, respectively, and $N_{\text {line }}^{\mathrm{AC}}$ and $N_{\text {line }}^{\mathrm{DC}}$ are the total numbers of lines in AC and DC networks, respectively.

The active and reactive power of the network lines are obtained using the existing power flow calculation method. In the optimization process, the node voltage amplitude constraint of the network is considered to be as in Equation (4).

$$
V_{s, \min }-\varepsilon_{1, s, t} \leq V_{s, t} \leq V_{s, \max }+\varepsilon_{2, s, t}, s \in N_{\text {node }}
$$

where $N_{\text {node }}$ is total network node of the system. $\varepsilon_{1, s, t}$ and $\varepsilon_{2, s, t}$ are relaxation variables. In practical calculation, voltage constraints are added as penalty terms to the objective function to avoid the occurrence of voltage over-limit conditions.

In this model, the flexible loads are mainly transferable loads (TLs), which can be expressed as Equation (5).

$$
\left\{\begin{array}{l}
\sum_{t=1}^{T} \alpha_{j, t}=X_{j} \\
P_{\mathrm{TL} j, t}=P_{\mathrm{TL}, j} \cdot \alpha_{j, t}, j \in N_{\mathrm{TL}}
\end{array}\right.
$$

where $\alpha_{j, t} \in\{0,1\}$ are the actual operating state of transferable load $j$ at time $t, 1$ is operation and 0 is outage. $N_{\mathrm{TL}}$ is the total number of transferable loads in the system.

Equation (5) is the constraint condition for the flexible load scheduling. Through the upper-level optimization, the flexible load scheduling result is obtained. Then it is passed to the lower level. Thus, the actual loads of DC and AC sections can be expressed as Equation (6).

$$
\left\{\begin{array}{l}
P_{\mathrm{L}_{-} \mathrm{DC}, t}=P_{\mathrm{CL} \_\mathrm{DC}, t}+\sum_{j_{1} \in N_{T L} \mathrm{DC} C} P_{T L_{-} D C j, t} \\
P_{\mathrm{L}_{-} \mathrm{AC}, t}=P_{\mathrm{CL} \_\mathrm{AC}, t}+\sum_{j_{2} \in N_{T L_{-} A C}} P_{T L_{-} A C j, t}
\end{array}\right.
$$

\subsection{Optimization Model of the Lower Level}

The lower-level optimization is divided into two parts: the DC section and the AC section, which have their own optimization objectives. As different stakeholders, they are committed to minimizing operating costs in their respective section.

\subsubsection{Optimization Model of the DC Section}

For the DC section, the scheduling costs mainly include the maintenance cost of generators and energy storage, the fuel cost of FC and CAES, and the electricity transaction cost. The objective function is shown in Equation (7).

$$
\min F_{11 \_D C}=\sum_{t=1}^{T}\left(C_{P V, t}+C_{W T, t}+C_{C A E S, t}+C_{F C, t}+C_{I L C \_D C, t}\right)
$$

The detailed expressions of the costs are expressed in Equation (8). 


$$
\left\{\begin{array}{l}
C_{\mathrm{PV}, t}=\sum_{i_{P V} \in N_{P V}}\left[c_{\mathrm{O}, P V} P_{P V, i_{P V}, t}+c_{a b a n, P V}\left(\bar{P}_{P V, i_{P V}, t}-P_{P V, i_{P V}, t}\right)\right] \Delta t \\
C_{\mathrm{WT}, t}=\sum_{i_{W T} \in N_{W T}}\left[c_{\mathrm{O}, W T} P_{W T, i_{W T}, t}+c_{a b a n, W T}\left(\bar{P}_{W T, i_{W T}, t}-P_{W T, i_{W T}, t}\right)\right] \Delta t \\
C_{\mathrm{CAES}, t}=\sum_{i_{C A E S} \in N_{C A E S}}\left(c_{C A E S} P_{\mathrm{CAES}, i_{C A E S, t}} u_{C A E S \_\mathrm{G}, t}+c_{\mathrm{o}, \mathrm{CAES}} P_{\left.\mathrm{CAES}, i_{C A E S, t}\right) \Delta t}\right. \\
C_{\mathrm{FC}, t}=\sum_{i_{F C} \in N_{F C}}\left(c_{F C} P_{F C, i_{F C, t}}+c_{\mathrm{O}, F C} P_{F C, i_{F C, t}}\right) \Delta t \\
C_{\mathrm{ILC} \_\mathrm{DC}, t}=c_{g, t} P_{\mathrm{ILC} \_\mathrm{DC}, t} \Delta t
\end{array}\right.
$$

where $N_{\mathrm{PV}}, N_{\mathrm{WT}}, N_{\mathrm{CAES}}, N_{\mathrm{FC}}$ are the total number of PV, WT, CAES and FC in the system. The second items in the right of $C_{\mathrm{PV}, t}$ and $C_{\mathrm{WT}, t}$ are the penalty cost of $\mathrm{PV}$ and wind power curtailment. These can ensure that the power of PV and WT is curtailed as little as possible.

Since CAES that has been applied on a large scale at this stage is mostly supplementarily fired, it needs to consume natural gas to supplement combustion during discharge stage. This cost has been considered in Equation (8). The simplified power model of CAES can be described as Equation (9).

$$
\left\{\begin{array}{l}
\dot{p}_{\text {CAES_C }, t}=k_{\text {CAES_C }} P_{\text {CAES_C }, t} \\
\dot{p}_{\text {CAES_G }, t}=k_{\text {CAES_G }} P_{\text {CAES_G }, t}
\end{array}\right.
$$

where $k_{\text {CAES_C }}$ and $k_{\text {CAES_G }}$ are the operation coefficients for compression and generation conditions, respectively, and their detailed expressions are presented in literature [29].

As CAES usually uses the working pressure of the gas storage chamber to indicate its charge and discharge capacity, the equivalent state of charge (SOC) can be described as Equation (10).

$$
p_{\mathrm{CAES}, t}=p_{\mathrm{CAES}, t-1}+u_{\mathrm{CAES} \_\mathrm{C}, t} k_{\mathrm{CAES} \_\mathrm{C}} P_{\mathrm{CAES} \_\mathrm{C}, t} \Delta t-u_{\mathrm{CAES} \_\mathrm{G}, t} k_{\mathrm{CAES} \_\mathrm{G}} P_{\mathrm{CAES} \_\mathrm{G}, t} \Delta t
$$

where $u_{\text {CAES_C }, t} \in[0,1]$ and $u_{\text {CAES_G, } t} \in[0,1]$ are the charge and discharge status at time $t$, respectively.

Thus, the constraints of CAES are shown in Equations (11)-(13).

$$
\begin{aligned}
& \left\{\begin{array}{l}
P_{\text {CASE_G,min }} \leq P_{\text {CASE_G }, t} \leq P_{\text {CAES_G, max }} \\
P_{\text {CAES_C } \min } \leq P_{\text {CAES_C } t} \leq P_{\text {CAES_C } m a x}
\end{array}\right. \\
& p_{\mathrm{CAES}, \min } \leq p_{\mathrm{CAES}, t} \leq p_{\mathrm{CAES}, \max } \\
& u_{\text {CAES_C }, t}+u_{\text {CAES_G }, t} \leq 1 \\
& p_{\mathrm{CAES}}(T)=p_{\mathrm{CAES}}(0)
\end{aligned}
$$

Equation (11) is the power constraint of CAES for the charge and discharge stages. Equation (12) is the equivalent SOC constraint of CAES. Equation (13) is the charge and discharge status constraint of CAES; this constraint ensures that CAES can only be charging, discharging or in a stop state at any given time. Equation (14) is the equivalent SOC balance constraint of CAES; this constraint ensures that CAES maintains the pressure of the gas storage chamber before and after a scheduling cycle.

The remaining constraints are shown in Equations (15)-(17).

$$
\begin{gathered}
\sum_{i_{P V} \in N_{P V}} P_{P V, i_{P V}, t}+\sum_{i_{W T} \in N_{W T}} P_{W T, i_{W T}, t}+\sum_{i_{C A E S} \in N_{C A E S}} P_{C A E S, i_{C A E S, t}}+\sum_{i_{F C} \in N_{F C}} P_{F C, i_{F C, t}}+P_{\mathrm{ILC} \_\mathrm{DC}, t}=P_{\mathrm{L} \_\mathrm{DC}, t} \\
P_{\mathrm{FC} \min , i_{\mathrm{FC}}} \leq P_{\mathrm{FC}, i_{\mathrm{FC}, t}} \leq P_{\mathrm{FCmax}, i_{\mathrm{FC}}} \\
P_{\mathrm{ILC} \_\mathrm{DC}, \min } \leq P_{\mathrm{ILC} \_\mathrm{DC}, t} \leq P_{\mathrm{ILC} \_\mathrm{DC}, \max }
\end{gathered}
$$

where Equation (15) is the power balance constraint of the DC section. Equation (16) is the output power constraint of the FC. Equation (17) is the output power constraint of the ILC. 


\subsubsection{Optimization Model of the AC Section}

Similarly, the objective function of the AC section is shown in Equation (18).

$$
\min F_{11 \_\mathrm{AC}}=\sum_{t=1}^{T}\left(c_{\mathrm{g}, t} P_{U P G, t}+c_{M T} P_{M T, i_{F C, t}}+c_{M T_{-} \mathrm{o}} P_{M T, i_{M T, t}}+c_{\mathrm{g}, t} P_{I L C_{-} A C, t}\right) \Delta t
$$

The constraints are shown in Equations (19)-(22).

$$
\begin{gathered}
P_{\mathrm{UPG}, t}+\sum_{i_{\mathrm{MT}} \in N_{\mathrm{MT}}} P_{\mathrm{MT}, i_{\mathrm{MT},} t}+P_{\mathrm{ILC} \_\mathrm{AC}, t}=P_{\mathrm{L} \_\mathrm{AC}, t} \\
P_{\mathrm{UPG}, \min } \leq P_{\mathrm{UPG}, t} \leq P_{\mathrm{UPG}, \mathrm{max}} \\
P_{\mathrm{MTmin}, i_{\mathrm{MT}}} \leq P_{\mathrm{MT}, i_{\mathrm{MT}, t}} \leq P_{\mathrm{MTmax}, i_{\mathrm{MT}}} \\
P_{\mathrm{ILC} \_\mathrm{AC}, t}=-P_{\mathrm{ILC} \_\mathrm{DC}, t}
\end{gathered}
$$

Equation (19) is the power balance constraint of the AC section, Equations (20) and (21) are the power constraints of UPG and MT respectively. According to the positive power direction shown in Figure 1, Equation (22) ensures the power balance of both the DC and AC sections.

Thus, the hierarchical scheduling model is established. It is summarized in Equation (23).

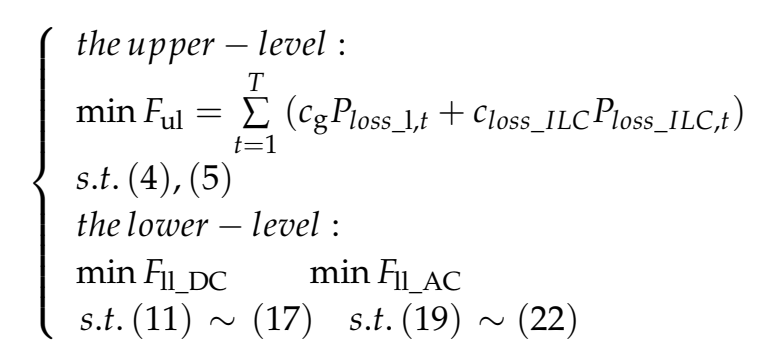

\section{Model Solution}

The optimization model of the AC/DC hybrid ADN is a bi-level optimization problem. The'network loss information in the upper-level optimization needs to be obtained according to the lower-level optimization result. Conversely, the lower-level optimization requires the output information of flexible loads determined in the upper-level optimization. Based on existing research, this chapter will present a DWDO algorithm, and combine it with the YALMIP toolkit to solve the bi-level optimization problem.

\subsection{Discrete Wind-Driven Optimization Algorithm}

Through the treatment of flexible loads in this paper, the upper-level optimization is a nonlinear integer optimization problem. At present, most nonlinear optimization problems are solved by intelligent algorithms, such as particle swarm optimization (PSO), genetic algorithm (GA), and so on. However, most of them have the problems that they find it easy to fall into local optima and are computationally inefficient. Thus, a wind-driven optimization (WDO) technique was proposed by [30], which is also an iterative heuristic global optimization algorithm. WDO is used to describe the motion of air parcels within the earth's atmosphere, and Newton's second law of motion is used to describe the N-dimensional search space. As a conclusion, [31] points out that WDO is well-suited for problems with both discrete and continuous-valued parameters. The algorithm process of WDO is as follows.

(1) Initialize the population size, set the maximum number of iterations, related parameters, and search boundaries, and define pressure functions.

(2) Initialize the air mass points and assign the starting speeds and locations randomly. 
(3) Calculate the pressure value of the air particles in the current iteration and rearrange the population according to the pressure values.

(4) Update the speeds of the air particles using Equation (24).

$$
\boldsymbol{u}_{\mathrm{new}}=(1-\alpha) \boldsymbol{u}_{\mathrm{cur}}-g \boldsymbol{x}_{\mathrm{cur}}+\left[R T\left|\frac{1}{i}-1\right|\left(\boldsymbol{x}_{\mathrm{opt}}-\boldsymbol{x}_{\mathrm{cur}}\right)\right]+\left(\frac{c \boldsymbol{u}_{\mathrm{cur}}^{\mathrm{otherdim}}}{i}\right)
$$

where $\boldsymbol{u}_{\text {new }}$ is the updated speed vector of air mass points, $\boldsymbol{u}_{\text {cur }}$ is current speed vector, $\boldsymbol{x}_{\text {cur }}$ is current location vector, $x_{\text {opt }}$ is the global optimum location so far, and $\boldsymbol{u}_{\text {cur }}^{\text {otherdim }}$ is the speed vector of any other dimension. $i$ is the arrangement number of the air mass points in step 3. $\alpha, g, R T$ and $c$ are parameters which will be changed according to the optimization problems.

(5) Update the positions of the air particles using Equation (25).

$$
x_{\text {new }}=x_{\text {cur }}+\left(u_{\text {new }} \Delta t\right)
$$

where $x_{\text {new }}$ is the updated location vector of the air mass points.

(6) If the termination condition is not reached, go to step 3.

The purpose of the upper-level optimization is to determine the output state of the flexible loads at each time. This is a discrete nonlinear optimization problem, which cannot be solved directly using the WDO algorithm. Therefore, the original WDO algorithm needs to be discretized, which is called DWDO in this paper. In DWDO, the starting position parameters should be randomly initialized as $0-1$ integer variables. The speed update formula is the same as Equation (24), but the positions need to be updated based on the probability of taking 1 . Now, introduce the sigmoid function, which is shown in Equation (26).

$$
s\left(\boldsymbol{u}_{\text {new }}\right)=\frac{1}{1+\exp \left(-\boldsymbol{u}_{\text {new }}\right)}
$$

In this place, $s\left(\boldsymbol{u}_{\text {new }}\right)$ indicates the probability of taking 1 for $x_{\text {new }}$. So the locations of the air particles can be updated by Equation (27).

$$
x_{\text {new }}= \begin{cases}1 & \text { if } \operatorname{rand}() \leq s\left(\boldsymbol{u}_{\text {new }}\right) \\ 0 & \text { otherwise }\end{cases}
$$

The remaining optimization steps of DWDO are similar to the original WDO algorithm. Using the DWDO algorithm, the upper-level optimization can obtain the optimized output for flexible loads.

\subsection{Solution Process of the Hierarchical Scheduling Model}

The lower-level optimization is a mixed-integer linear programming (MILP) problem. As a mature optimization toolkit, YALMIP has great advantages in solving MILP problems, and has been widely used [32-34]. Furthermore, it can easily interact with MATLAB. Therefore, YALMIP is used to solve lower-level optimization and iterates with the DWDO algorithm in the upper-level optimization. The solution process is shown in Figure 3.

As this paper focuses on active economic dispatch of ADN, only active power is allowed to be exchanged between the ADN and the UPG. The DC and AC sections exchange active power through ILC. In addition to this, the AC section also exchanges active power with the UPG through the boundary line. Reactive power is generated locally by suitable means to satisfy the reactive demand and losses for both DC and AC sections. Thus, the solution process of the hierarchical scheduling model is as follows.

(1) Initialize the values of $P_{\mathrm{CAES}}, P_{\mathrm{FC}}, P_{\mathrm{MT}}, P_{\mathrm{ILC}}$ using operation data of another similar day, and preset the planned output of the flexible loads.

(2) Solve the upper-level optimization using DWDO, in order to obtain the optimized output of the flexible loads ( $P_{\mathrm{TL} \_\mathrm{DC}}^{*}, P_{\mathrm{TL} \_\mathrm{AC}}^{*}$ ). In this process, we expect that $\mathrm{PV}$ and wind power can be 
absorbed as much as possible. In addition, as the AC side is connected to the upper-level power grid, its internal power adjustment ability is strong. Therefore, the optimization starts from the DC side in this step.

(3) Solve the optimization of the DC and AC sections using YALMIP, in order to obtain $P_{\mathrm{CAES}}^{*} P_{\mathrm{FC}}^{*}$ $P_{\mathrm{ILC}}^{*} P_{\mathrm{MT}}^{*}$.

(4) Determine whether the algorithm reaches a defined number of iterations or convergence accuracy. If the change of the system cost is lower than a certain constant between two iterations, the algorithm converges and then the result is presented. If not, go to step 2.

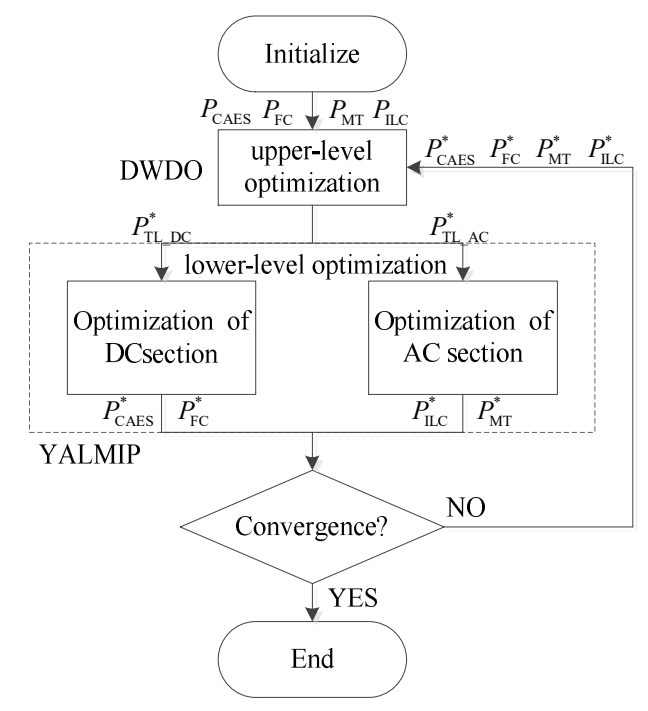

Figure 3. Solution process of the hierarchical scheduling model.

\section{Case Study and Discussion}

The hierarchical scheduling model is tested in a modified 38-node test system, which is shown in Figure 4. The modified test system is divided into a DC section and an AC section. The DC section includes two PVs, one WT, one FC and one CAES, while the AC section includes one MT and is connected to the upper-level power grid. The configuration of loads is also shown in Figure 4. The line parameters are given in literature [35].

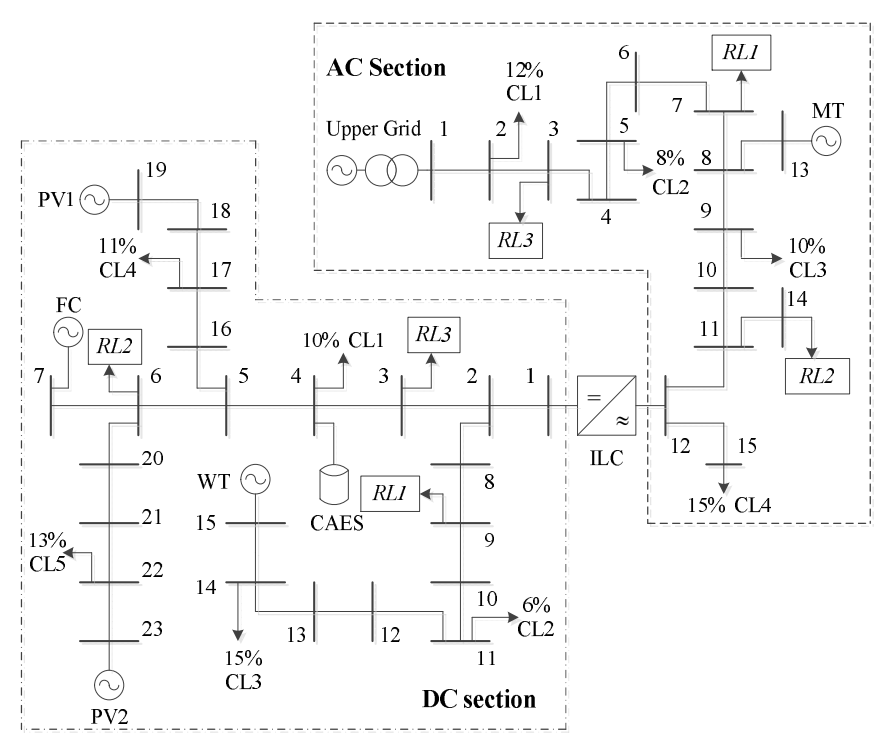

Figure 4. Modified 38-node test system. 
In this system, the forecast output of the PVs and WT is shown in Figure 5, while the total rigid load prediction curve of the whole system is shown in Figure 6. The data for transferable loads is shown in Table 1. The data for CAES is shown in Table 2. The time-of-use price (TOU) of UPG is shown in Table 3. The maintenance cost of the PVs, WT, FC and MT are $0.0096 \mathrm{CNY} / \mathrm{kWh}$, $0.0296 \mathrm{CNY} / \mathrm{kWh}, 0.088 \mathrm{CNY} / \mathrm{kWh}$ and $0.088 \mathrm{CNY} / \mathrm{kWh}$, respectively. The fuel cost of the FC and MT are $0.525 \mathrm{CNY} / \mathrm{kWh}$ and $0.641 \mathrm{CNY} / \mathrm{kWh}$, respectively. The penalty cost coefficients of $\mathrm{PV}$ and wind power curtailment is $10 \mathrm{CNY} / \mathrm{kWh}$. The power limits of the ILC and the UPG are $\pm 1000 \mathrm{~kW}$ and $\pm 1500 \mathrm{~kW}$, respectively.

The parameters of DWDO are $\alpha=0.4, g=0.2, R T=3, c=0.4$. The number of air mass points is 30 , the number of iterations is 500. All programs are coded and tested in MATLAB, and the lower-level optimization is solved by YALMIP. The programs run on an Intel(R) Core(TM) i7-4710MQ @ $2.50 \mathrm{GHz}$, RAM 8 GB system.

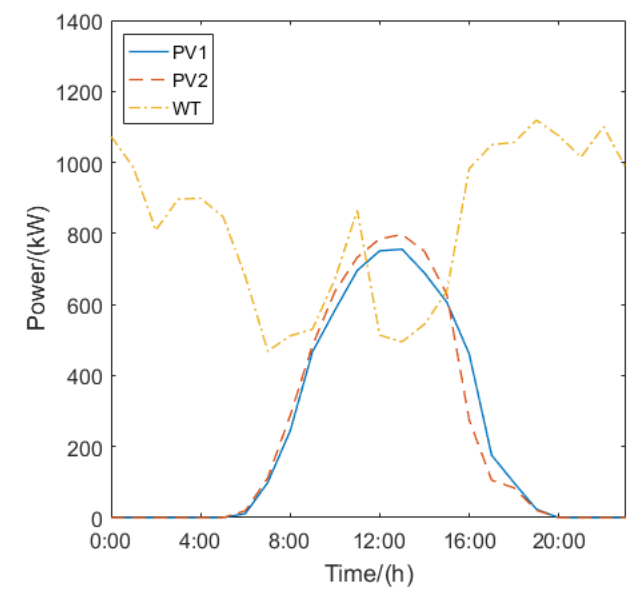

Figure 5. Prediction curve of PVs and WT.

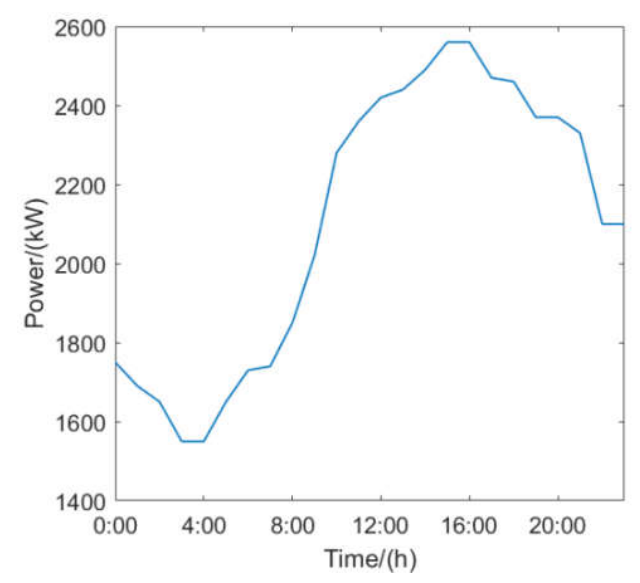

Figure 6. Prediction curve of rigid load.

Table 1. Data of transferable loads.

\begin{tabular}{cccc}
\hline Transferable Loads & Rated Power (Kw) & Planned Operation Time (h) & Planned Outage Time (h) \\
\hline RL1 of AC section & 180 & $9-12,15-18,22-24$ & $0-8,13-17,19-21$ \\
RL2 of AC section & 200 & $11-14,19-21$ & $0-10,15-18,22-24$ \\
RL3 of AC section & 160 & $0-18$ & $19-24$ \\
RL1 of DC section & 180 & $8-17$ & $0-7,18-24$ \\
RL2 of DC section & 240 & $6-8,11-13,18-22$ & $0-5,9-10,14-17,23-24$ \\
RL3 of DC section & 200 & $5-22$ & $0-4,23-24$ \\
\hline
\end{tabular}


Table 2. Data for CAES.

\begin{tabular}{cc}
\hline Item & Value \\
\hline Rated discharge power $(\mathrm{kW})$ & 1000 \\
Rated charge power $(\mathrm{kW})$ & 700 \\
Initial pressure (bar) & 55 \\
Cavern operational pressure range $(\mathrm{bar})$ & $46-66$ \\
Operation coefficient for compression $(\mathrm{bar} / \mathrm{kW})$ & 0.0071 \\
Operation coefficient for generation $(\mathrm{bar} / \mathrm{kW})$ & 0.0033 \\
Power generation cost $(\mathrm{CNY} / \mathrm{kW})$ & 0.16 \\
Maintenance cost $(\mathrm{CNY} / \mathrm{kW})$ & 0.0013 \\
\hline
\end{tabular}

Table 3. Time-of-use price of UPG.

\begin{tabular}{cc}
\hline Time (h) & Price (CNY/kWh) \\
\hline $0-7$ & 0.3 \\
$7-12$ & 0.6 \\
$12-14$ & 1 \\
$14-18$ & 0.6 \\
$18-22$ & 1 \\
$22-24$ & 0.3 \\
\hline
\end{tabular}

To analyze the effectiveness of the proposed hierarchical scheduling strategy, we consider the following two case studies:

CASE 1: Hierarchical optimization of two levels, but the lower-level is optimized as a whole.

CASE 2: Hierarchical optimization of two levels, and the lower-level is divided into two stakeholders.

\subsection{Economic Comparison}

CASE 1: This case is the conventional scheduling strategy that already exists. In this case, the AC/DC hybrid ADN is regarded as one stakeholder, and the ILC power limitation is not considered. The scheduling result is shown in Figure 7. In the depth of the night, the CAES charges, as both the system load and the electricity price of the UPG are low. At noon, from 12:00 to 14:00, the outputs of the PVs are high, and the power supply for the ADN is sufficient. The electricity price of the UPG reaches its maximum, which is higher than the MT and FC. Therefore, all DGs run in order to sell electricity to the UPG in order to gain substantial profits. At 15:00-17:00, although the rigid load of the system reaches peak value, the power of the PVs and WT is able to meet most load requirements. At 18:00-21:00, the electricity price of the UPG reaches maximum again. Now the outputs of the PVs are almost zero, and the FC and MT are running to sell electricity to the UPG. However, at this time, the sales power is less than that at noon. As a result, the operating costs of the $\mathrm{AC}$ and $\mathrm{DC}$ sections are $16,764 \mathrm{CNY}$ and $-2967 \mathrm{CNY}$ respectively, and the cost of system loss is $4651 \mathrm{CNY}$.

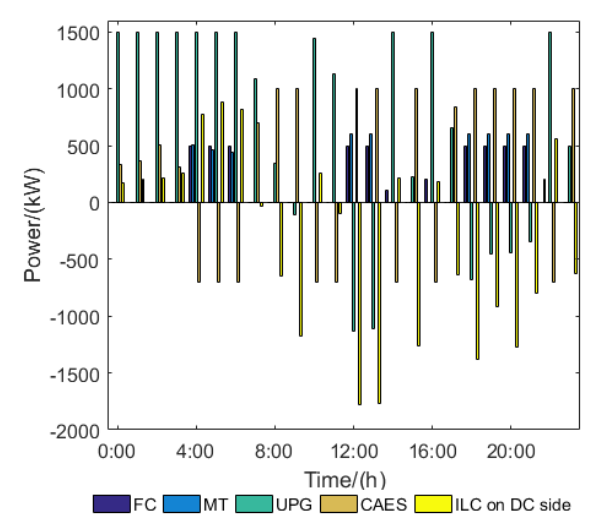

Figure 7. Scheduling result of CASE 1. 
CASE 2: This case is the proposed hierarchical scheduling strategy in this paper. The lower level is divided into two stakeholders, and the transmission power limit between the two stakeholders is taken into account. The scheduling result is shown in Figure 8. Compared to CASE 1, the ADN will also sell electricity to the UPG at 12:00-14:00 and 18:00-21:00 in CASE 2, but the sales power is less than CASE 1. As a result, the operating costs of the AC and DC sections are 16,673 CNY and $2580 \mathrm{CNY}$, respectively, and the cost of the system loss is 4643 CNY. Obviously, the total cost in CASE 2 is more than that in CASE 1, due to the transmission power limitation of ILC. From Figure 7, we know that the maximum transmission power of ILC is about $1800 \mathrm{~kW}$, which is far more than the $1000 \mathrm{~kW}$ in CASE 2. This means more investment is needed in the construction of the distribution network. Thus, it is inappropriate to regard the AC/DC hybrid ADN as one stakeholder without considering the constraints between the AC and DC sub-networks.

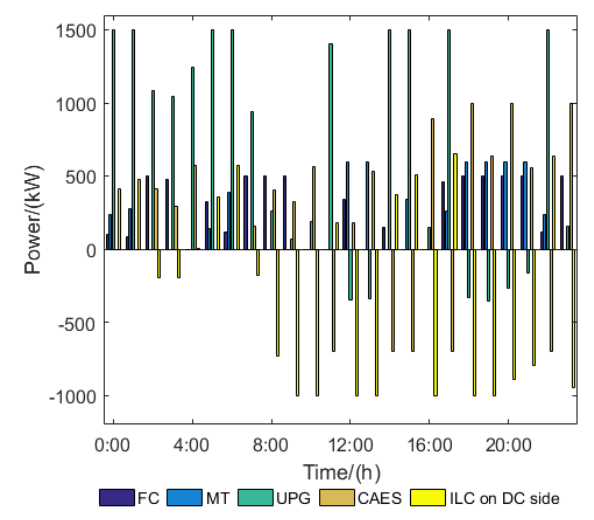

Figure 8. Scheduling result of CASE 2.

\subsection{Scheduling Result of Loads}

The scheduling result of the loads in CASE 2 is shown in Figure 9. The planned loads are low at 0:00-6:00 and high at 15:00-17:00. This may lead to excess or insufficient power supply for the ADN. By means of the optimization scheduling, flexible loads are transferred to achieve peak load shifting. The scheduled load curve is more stable, and the load peak-to-valley difference is smaller.

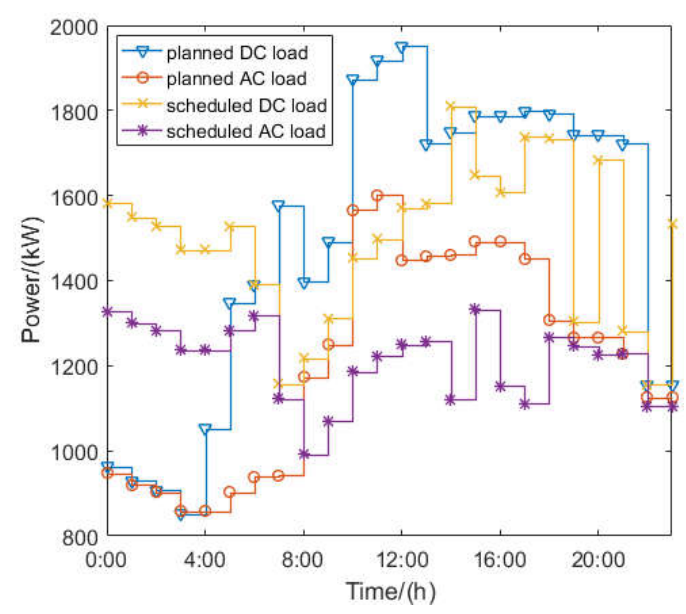

Figure 9. Load scheduling result of CASE 2.

\subsection{ILC Power Analysis}

The power balance of ILC is important in the optimization process. Usually, in optimization scheduling, decision-makers mainly focus on the active power. The power profiles of ILC on the DC side and the AC side are shown in Figure 10. According to the previous analysis, we know that a large amount of load was transferred to 0:00-2:00. Now, the electricity price of UPG is low and the 
AC sub-network buys electricity from the UPG. Part of the purchased electricity is transmitted to the DC sub-network through the ILC. Between around 10:00 and 12:00, the outputs of WT and PVs are high, and ILC delivers power from the DC sub-network to the AC sub-network at maximum power. At 18:00-21:00, the electricity price of UPG is high, and the output of WT is high. Thus, power is also delivered from the DC sub-network to the AC sub-network during this time. It can be seen from Figure 10 that the power of the ILC on the AC and DC side is balanced in a scheduling period.

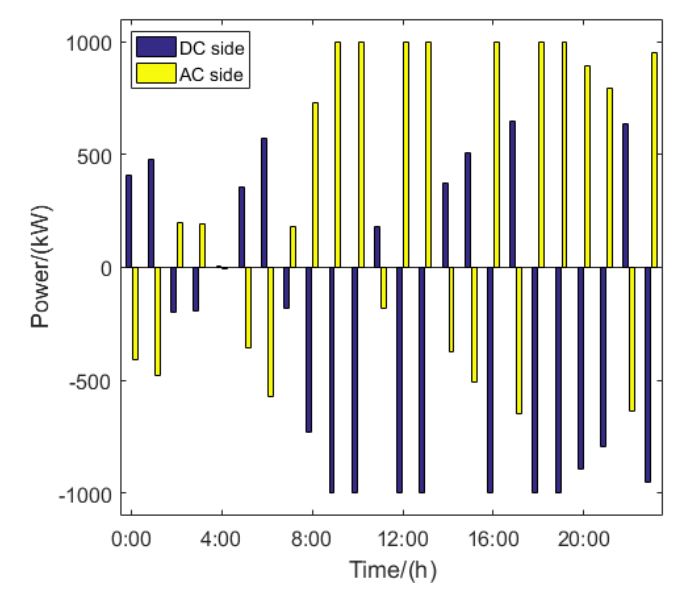

Figure 10. Power of ILC on both the DC and the AC side.

\subsection{Rejected Power Analysis}

Due to the existence of system constraints, the active power may be rejected by other stakeholders. The actual output powers of WT and PVs are shown in Figure 11. The actual outputs of PVs are the same as the prediction curve, but there is a reduction in the power of WT. In a scheduling period, the maximum total output of wind power is $19,811 \mathrm{kWh}$, but the total output is actually $19,501 \mathrm{kWh}$. The transmission power constraints of ILC and UPG will affect it, as well as the capacity of CAES.

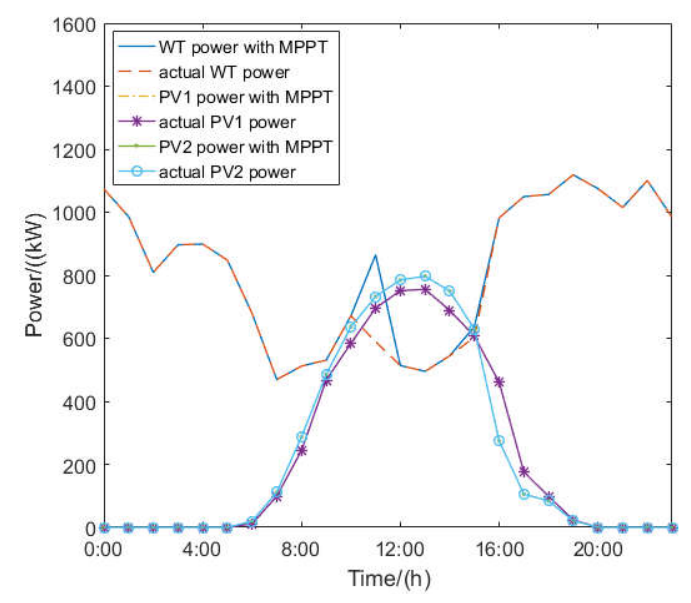

Figure 11. Actual output power and MPPT power of WT, PV1 and PV2.

For the DC section, the rejected power of WT and PVs is mainly related to the ILC power limitation and the capacity of the energy storage system. Meanwhile, for the whole AC/DC hybrid ADN, the rejected power is mainly affected by the power limitation of the boundary line with UPG. For instance, when the transmission power limit of the UPG is increased to $3000 \mathrm{~kW}$, no rejected power will appear. Detailed quantitative analysis will be carried out in future research. 


\section{Conclusions and Prospects}

As an $\mathrm{AC} / \mathrm{DC}$ hybrid, $\mathrm{ADN}$ is becoming an important implementation forms of the future distribution network, its global optimization scheduling is of great significance. For this issue, a hierarchical multi-stage scheduling model is established in this paper. The upper level optimizes the total system losses by scheduling the flexible loads, while the lower level minimizes the scheduling costs of the AC and DC sections by scheduling the controllable DGs and CASE. The DWDO algorithm and the YALMIP toolbox are combined to solve the scheduling model. The case study shows that the proposed scheduling scheme can achieve lower system loss with low operating costs of both AC and DC sections. Thus, not only is the overall system optimized, but the profits of multi-stakeholders are also taken into account. The research in this paper can provide several theoretical references for the scheduling of the AC/DC hybrid distribution network.

Author Contributions: C.Y. and S.M. conceived and designed the study. C.Y., C.L. and Y.L. carried out the simulation. C.Y., S.M. and L.L. analyzed simulation results. C.Y., C.L. and Y.L. wrote the paper. C.Y., S.M. and L.L. reviewed and edited the manuscript. All authors read and approved the manuscript.

Funding: This research was funded by National Key Research and Development Program of China (2017YFB0903601), The National Natural Science Foundation of China (51777088) and 2018 scientific research project of State Grid Corporation of China (SGNXDK00DWJS1800016).

Conflicts of Interest: The authors declare no conflict of interest.

\section{Nomenclature}

\begin{tabular}{|c|c|}
\hline$F_{\mathrm{ul}}$ & the upper-level optimization objective \\
\hline$P_{\text {loss }, l, t}$ & the line loss of the entire network at time $t$ \\
\hline$P_{\text {loss_ILC, } t}$ & the active power loss of ILC at time $t$ \\
\hline$c_{\mathrm{g}, t}$ & the time-of-use price of UPG at time $t$ \\
\hline$c_{\text {loss_ILC }}$ & the cost of the ILC loss \\
\hline$P_{\text {loss }, l_{1}, t}, P_{\text {loss }, l_{2}, t}$ & line losses of $\mathrm{AC}$ and $\mathrm{DC}$ network respectively at time $t$ \\
\hline$P_{l_{1}, t}, Q_{l_{1}, t}$ & active and reactive power of line $l_{1}$ at time $t$ \\
\hline$P_{l_{2}, t}$ & active power of line $l_{2}$ at time $t$ \\
\hline$R_{l_{1}}, R_{l_{2}}$ & line resistances of $l_{1}$ and $l_{2}$ \\
\hline$U_{\mathrm{e} \_\mathrm{AC}}, U_{\mathrm{e} \_\mathrm{DC}}$ & rated voltages of $\mathrm{AC}$ and $\mathrm{DC}$ buses \\
\hline$P_{\mathrm{ILC}, t}$ & transmission power of ILC at time $t$ \\
\hline$\eta_{\text {ILC }}$ & effectiveness of ILC \\
\hline$V_{s, t}$ & voltage of node $s$ \\
\hline$V_{s, \max }, V_{s, \min }$ & maximum and minimum allowable voltage of node $s$ \\
\hline$X_{j}$ & total operating time in a scheduling period of transferable load $j$ \\
\hline$P_{\mathrm{TL}, j}$ & rated power of transferable load \\
\hline$P_{\mathrm{TL} j, t}$ & actual power of transferable load at time $t$ \\
\hline$P_{\mathrm{L} \_\mathrm{DC}, t}, P_{\mathrm{L} \_\mathrm{AC}, t}$ & total load of DC and AC section at time $t$ \\
\hline$P_{\mathrm{CL} \_\mathrm{DC}, t}, P_{\mathrm{CL} \_\mathrm{AC}, t}$ & constant load of DC and AC section at time $t$ \\
\hline$P_{\mathrm{TL} \_\mathrm{DC}, t}, P_{\mathrm{TL} \_\mathrm{AC}, t}$ & transferable load of DC and AC section at time $t$ \\
\hline$N_{\mathrm{TL} \_\mathrm{DC}}, N_{\mathrm{TL} \_\mathrm{AC}}$ & total number of transferable loads in DC and AC section \\
\hline$C_{\mathrm{PV}}, C_{\mathrm{WT}}, C_{\mathrm{CAES}}, C_{\mathrm{FC}}$ & operating cost of PV, WT, CAES and FC \\
\hline$c_{\mathrm{aban}, \mathrm{PV}}, c_{\mathrm{aban}, \mathrm{WT}}$ & penalty cost coefficients of PV and wind power curtailment \\
\hline $\bar{P}_{\mathrm{PV}, i_{\mathrm{PV}}, t}, \bar{P}_{\mathrm{WT}, i_{\mathrm{WT}}, t}$ & output power of PV and WT with maximum power point tracking (MPPT) \\
\hline C & electricity transaction cost which is carried out through ILC on DC side \\
\hline$c_{\mathrm{O}, \mathrm{PV}}, c_{\mathrm{O}, \mathrm{PV}}, c_{\mathrm{O}, \mathrm{CAES}}, c_{\mathrm{O}, \mathrm{FC}}$ & maintenance cost coefficient of PV, WT, CAES and FC \\
\hline$c_{\mathrm{CAES}}, c_{\mathrm{FC}}$ & power generation cost coefficient of CAES and FC \\
\hline$P_{\mathrm{PV}, t}, P_{\mathrm{WT}, t}, P_{\mathrm{CAES}, t}, P_{\mathrm{FC}, t}$ & actual output power of PV, WT, CAES and FC \\
\hline$P_{\text {ILC_DC }, t}$ & power of ILC on DC side \\
\hline$\dot{p}_{\text {CAES_C }, t}, \dot{p}_{\text {CAES_G }, t}$ & rate of pressure change for compression and generation conditions \\
\hline
\end{tabular}




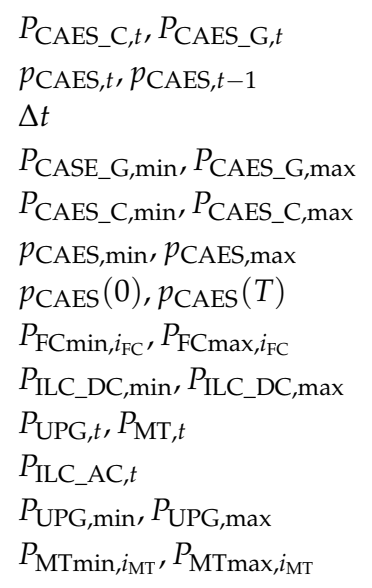

$P_{\text {CAES_C, } t}, P_{\text {CAES_G }, t}$

$\Delta t$

$P_{\text {CASE_G, min }}, P_{\text {CAES_G, max }}$

$P_{\text {CAES_C,min }}, P_{\text {CAES_C, max }}$

$p_{\mathrm{CAES}, \mathrm{min}}, p_{\mathrm{CAES}, \mathrm{max}}$

$p_{\text {CAES }}(0), p_{\text {CAES }}(T)$

$P_{\mathrm{FCmin}, i_{\mathrm{FC}}}, P_{\mathrm{FCmax}, i_{\mathrm{FC}}}$

$P_{\text {ILC_DC, } \min }, P_{\text {ILC_DC, } \max }$

$P_{\mathrm{UPG}, t}, P_{\mathrm{MT}, t}$

$P_{\mathrm{UPG}, \min }, P_{\mathrm{UPG}, \max }$

\section{References}

\author{
power of CAES for compression and generation conditions \\ pressure of the gas storage chamber at time $t$ and time $t-1$ \\ interval between two scheduling time \\ minimum and maximum allowable discharge power of CAES \\ minimum and maximum allowable charge power of CAES \\ minimum and maximum allowable pressure of the gas storage chamber \\ pressure of the gas storage chamber at time 0 and time $T$ \\ minimum and maximum allowable output power of FC \\ minimum and maximum allowable transmission power of ILC \\ output power of UPG and MT at time $t$ \\ power of ILC on AC side \\ minimum and maximum allowable transmission power Of UPG \\ minimum and maximum allowable output power of MT
}

1. Huang, S.; Wu, Q.; Liu, Z.; Nielsen, A.H. Review of congestion management methods for distribution networks with high penetration of distributed energy resources. In Proceedings of the 5th IEEE PES Innovative Smart Grid Technologies Europe, Istanbul, Turkey, 12-15 October 2014.

2. Cipcigan, L.M.; Taylor, P.C. Investigation of the reverse power flow requirements of high penetrations of small-scale embedded generation. IET Renew. Power Gener. 2007, 1, 160-166. [CrossRef]

3. Gabash, A.; Xie, D.; Li, P. Analysis of influence factors on rejected active power from active distribution networks. In Proceedings of the IEEE Power and Energy Student Summit, Ilmenau, Germany, 19-20 January 2012.

4. Imani, M.H.; Zalzar, S.; Mosavi, A.; Shamshirband, S. Strategic behavior of retailers for risk reduction and profit increment via distributed generators and demand response programs. Energies 2018, 11, 1602. [CrossRef]

5. Liu, X.; Wang, P.; Loh, P.C. A hybrid AC/DC microgrid and its coordination control. IEEE Trans. Smart Grid 2011, 2, 278-286.

6. Kurohane, K.; Senjyu, T.; Uehara, A.; Yona, A.; Funabashi, T.; Kim, C.-H. A hybrid smart AC/DC power system. In Proceedings of the 5th IEEE Conference on Industrial Electronics and Applications, Taichung, Taiwan, 15-17 June 2010.

7. Eghtedarpour, N.; Farjah, E. Power control and management in a hybrid AC/DC microgrid. IEEE Trans. Smart Grid 2014, 5, 1494-1505. [CrossRef]

8. Wang, P.; Goel, L.; Liu, X.; Choo, F.H. Harmonizing AC and DC: A hybrid AC/DC future grid solution. IEEE Power Energy Mag. 2013, 11, 76-83. [CrossRef]

9. Unamuno, E.; Barrena, J.A. Hybrid ac/dc microgrids-Part I: Review and classification of topologies. Renew. Sustain. Energy Rev. 2015, 52, 1251-1259. [CrossRef]

10. Yang, Z.; Zhong, H.; Bose, A.; Xia, Q.; Kang, C. Optimal power flow in AC-DC grids with discrete control devices. IEEE Trans. Power Syst. 2018, 33, 1461-1472. [CrossRef]

11. Cao, J.; Du, W.; Wang, H.F. An improved corrective security constrained OPF for meshed AC/DC grids with multi-terminal VSC-HVDC. IEEE Trans. Power Syst. 2016, 31, 485-495. [CrossRef]

12. Baradar, M.; Hesamzadeh, M.R.; Ghandhari, M. Second-Order cone programming for optimal power flow in VSC-type AC-DC grids. IEEE Trans. Power Syst. 2013, 28, 4282-4291. [CrossRef]

13. Malik, S.M.; Ai, X.; Sun, Y.; Chen, Z.; Zhou, S. Voltage and frequency control strategies of hybrid AC/DC microgrid: A review. IET Gener. Transm. Distrib. 2017, 11, 303-313. [CrossRef]

14. Shanthi, P.; Govindarajan, U.; Parvathyshankar, D. Instantaneous power-based current control scheme for VAR compensation in hybrid AC/DC networks for smart grid applications. IET Power Electron. 2014, 7, 1216-1226. [CrossRef]

15. Dong, L.; Ming, J.; Yu, T.; Fan, S.; Pu, T. Voltage division control in AC / DC hybrid distribution network based on model predictive control. In Proceedings of the 2016 IEEE PES Asia-Pacific Power and Energy Engineering Conference (APPEEC), Xi'an, China, 25-28 October 2016.

16. Zhu, M.; Hang, L.; Li, G.; Jiang, X. Protected control method for power conversion interface under unbalanced operating conditions in AC/DC hybrid distributed grid. IEEE Trans. Energy Convers. 2016, 31, 57-68. [CrossRef] 
17. Nejabatkhah, F.; Li, Y. Overview of power management strategies of hybrid AC/DC microgrid. IEEE Trans. Power Electron. 2015, 30, 7072-7089. [CrossRef]

18. Liu, S.; Ding, L.; Miao, Y. Research of coordinated control strategy for multi-UHVDC in AC/DC hybrid power grid. Energy Procedia 2011, 12, 443-449. [CrossRef]

19. Hu, J.; Shan, Y.; Xu, Y.; Guerrero, J.M. A coordinated control of hybrid ac/dc microgrids with PV-wind-battery under variable generation and load conditions. Int. J. Electr. Power Energy Syst. 2019, 104, 583-592. [CrossRef]

20. Li, Y.; Li, Y.; Li, G.; Zhao, D.; Chen, C. Two-stage multi-objective OPF for AC/DC grids with VSC-HVDC: Incorporating decisions analysis into optimization process. Energy 2018, 147, 286-296. [CrossRef]

21. Ghadiri, A.; Haghifam, M.R.; Larimi, S.M.M. Comprehensive approach for hybrid AC/DC distribution network planning using genetic algorithm. IET Gener. Transm. Distrib. 2017, 11, 3892-3902. [CrossRef]

22. Liang, H.; Lin, J.; Ba, L.; Li, H. Research on optimal dispatch method for AC/DC hybrid active distribution network. In Proceedings of the International Conference on Renewable Power Generation (RPG), Beijing, China, 17-18 October 2015.

23. Indragandhi, V.; Logesh, R.; Subramaniyaswamy, V.; Vijayakumar, V.; Siarry, P.; Uden, L. Multi-objective optimization and energy management in renewable based AC/DC microgrid. Comput. Electr. Eng. 2018, 70, 179-198.

24. Qi, C.; Wang, K.; Li, G.; Han, B.; Xu, S.; Wei, Z. Hierarchical and distributed optimal scheduling of AC/DC hybrid active distribution network. Proc. CSEE 2017, 37, 1909-1917.

25. Eajal, A.A.; Shaaban, M.F.; Ponnambalam, K.; El-Saadany, E.F. Stochastic centralized dispatch scheme for AC/DC hybrid smart distribution systems. IEEE Trans. Sustain. Energy 2017, 7, 1046-1059. [CrossRef]

26. Qiu, H.; Zhao, B.; Gu, W.; Bo, R. Bi-Level two-stage robust optimal scheduling for AC/DC hybrid multi-microgrids. IEEE Trans. Smart Grid 2018, 9, 5455-5466. [CrossRef]

27. Qiu, H.; Gu, W.; Pan, J.; Xu, B.; Xu, Y.; Fan, M.; Wu, Z. Multi-interval-uncertainty constrained robust dispatch for AC/DC hybrid microgrids with dynamic energy storage degradation. Appl. Energy 2018, 228, 205-214. [CrossRef]

28. Gabash, A.; Li, P. On variable reverse power flow-part I: Active-reactive optimal power flow with reactive power of wind stations. Energies 2016, 9, 121. [CrossRef]

29. Li, Y.; Miao, S.; Luo, X.; Wang, J. Optimization model for the power system scheduling with wind generation and compressed air energy storage combination. In Proceedings of the 22nd International Conference on Automation and Computing (ICAC), Colchester, UK, 7-8 September 2016.

30. Bayraktar, Z.; Komurcu, M.; Werner, D.H. Wind Driven Optimization (WDO): A novel nature-inspired optimization algorithm and its application to electromagnetics. In Proceedings of the 2011 IEEE Antennas and Propagation Society International Symposium, Toronto, ON, Canada, 11-17 July 2010.

31. Bayraktar, Z.; Komurcu, M.; Bossard, J.A.; Werner, D.H. The wind driven optimization technique and its application in electromagnetics. IEEE Trans. Antennas Propag. 2013, 61, 2745-2757. [CrossRef]

32. Lofberg, J. YALMIP: A toolbox for modeling and optimization in MATLAB. In Proceedings of the 2004 IEEE International Conference on Robotics and Automation, New Orleans, LA, USA, 2-4 September 2004.

33. Kekatos, V.; Giannakis, G.B.; Wollenberg, B. Optimal placement of phasor measurement units via convex relaxation. IEEE Trans. Power Syst. 2012, 27, 1521-1530. [CrossRef]

34. Korres, G.N.; Manousakis, N.M.; Xygkis, T.C.; Löfberg, J. Optimal phasor measurement unit placement for numerical observability in the presence of conventional measurements using semi-definite programming. IET Gener. Transm. Distrib. 2015, 9, 2427-2436. [CrossRef]

35. Singh, D.; Misra, R.; Singh, D. Effect of load models in distributed generation planning. IEEE Trans. Power Syst. 2007, 22, 2204-2212. [CrossRef]

(C) 2018 by the authors. Licensee MDPI, Basel, Switzerland. This article is an open access article distributed under the terms and conditions of the Creative Commons Attribution (CC BY) license (http:/ / creativecommons.org/licenses/by/4.0/). 